\title{
Giemsa C-banding Karyotype and Detection of Polymorphic Constitutive Heterochromatin in Nigella sativa L.
}

\author{
Indranil Santra ${ }^{1}$, Sk Moquammel Haque ${ }^{1,2}$ and Biswajit Ghosh ${ }^{1 *}$ \\ ${ }^{1}$ Plant Biotechnology Laboratory, Post Graduate Department of Botany, Ramakrishna Mission Vivekananda \\ Centenary College, Rahara, Kolkata 700118, India \\ ${ }^{2}$ Department of Botany, East Calcutta Girls' College, Lake Town, Kolkata 700089, India
}

Received November 8, 2019; accepted November 28, 2019

\begin{abstract}
Summary Karyomorphology, distribution of constitutive heterochromatin, and intrapopulation polymorphism of C-bands in a cross-pollinated population of Nigella sativa L. were studied. Enzymatic digestion of the cell wall allows cytoplasm-free chromosome preparation and enhances the stain resolution, thereby promoting accurate chromosome measurements and identification of chromosomal landmarks. The diploid chromosome number is $2 n=12$, of which five pairs are moderately long and have median centromere, whereas one small pair has a subterminal centromere. Secondary constriction has been observed in two pairs of long chromosomes. Polymorphism of the C-band size in individuals of the same population and even between two homologous chromosomes of a single individual has been detected by the C-banding technique. Further, the absence of any DAPI-bands indicated that the DNA of C-band region is not AT-rich. The heterogeneity of C-bands in homologous pairs of a single individual is a consequence of cross-pollination where chromosomes come from two different parents. The pattern of heterochromatin distribution can serve as a unique marker that can enrich the karyosystematic database and prove useful in the breeding program of $N$. sativa.
\end{abstract}

Keywords C-bands, Constitutive heterochromatin, DAPI, Enzyme digestion, Karyotype, Nigella sativa.

Nigella sativa L. is an open-pollinated flowering plant belonging to the family Ranunculaceae, native to southwest Asia but widely cultivated in different parts of the world (Khan et al. 2011). The seeds of N. sativa have traditionally been used for thousands of years as a spice, food additive, and herbal medicine to cure diarrhea, headache, and toothache. The ability of $N$. sativa as an anti-proliferative, antimetastatic, and proapoptotic agent on various primary cancer cells has also been reported (Majdalawieh and Fayyad 2016). The worldwide demand for this crop has made it economically viable in the international market (Dubey et al. 2016).

Analysis of its chromosomal characters can provide basic genetic information, which can prove useful in the breeding of economically important crops (Sybenga 1992, Haque et al. 2016). The chromosomes of the genus Nigella are comparatively long and fewer in number; hence, they are considered to be fine material for cytological studies (Gilot-Delhalle et al. 1976, Marks 1977, Ghosh and Datta 2006, Shaker et al. 2017). Although karyological analysis was reported in $N$. sativa, only limited data is available for chromosome banding and fluorescent staining (Gilot-Delhalle et al. 1976, Ghosh and Datta 2006, Jabeen et al. 2012, Shaker et al. 2017).

\footnotetext{
*Corresponding author, e-mail: ghosh_b2000@yahoo.co.in DOI: $10.1508 /$ cytologia. 85.85
}

Application of chromosome banding and fluorescent staining in plant chromosome analysis has a significant value over conventional orcein-based karyotype studies.

Heterochromatin is an essential genetic element with several cytological and genetic attributes. Heterochromatin can be categorized into two types, namely, constitutive heterochromatin and facultative heterochromatin. Constitutive heterochromatin is commonly distributed in the centromeric, pericentromeric, and telomeric portion and can also be found in nucleolus organizer regions (NORs), suggesting their importance in chromosome organization, telomeric protection, and centromeric function. The C-banding technique in the plant system, introduced by Vosa and Marchi (1972), helps identify the presence and distribution of constitutive heterochromatin in the genome. Related groups of species have tendencies to show more or less similar C-banding patterns in their karyotype profile (Blakey and Vosa 1981).

Rising of polymorphic and polytypic populations due to the occurrence of variable constitutive heterochromatin contents at the intraspecific level is considered a general feature of eukaryotes (John 1988, DávilaRodríguez et al. 2011). Quantitative variation of the heterochromatin content in both plant and animal systems has been reported earlier (John 1981, Schwarzacher and Schweizer 1982). A geographically isolated population of Arabidopsis thaliana was found to exhibit a 
significant variation of heterochromatin content in their genome (Davison et al. 2007). Although the proper roles of heterochromatin are yet to be understood, it has been presumed that they play an essential part in chromosome stabilization (Verma 1998). In addition, they play some functional roles in chromosome pairing, segregation, recombination variegation, and position effect (John and Miklos 1979, Gatti and Pimpinelli 1992). Besides, C-banding is also essential to identify the intraspecific polymorphism in the heterochromatin content according to their amount and position, which might indicate the adaptive value of the species with their growing habitat (Vosa 1976).

Therefore, in this study, detailed karyomorphological analysis has been presented along with their Giemsa Cbanding pattern. The distribution pattern of C-bands can not only serve as a marker to identify different chromosomes of Nigella species but also improve the karyotype database, which can be exploited for plant breeding and agricultural purposes.

\section{Materials and methods}

Seeds of a cultivated population of Nigella sativa were collected from Nadia district $\left(88^{\circ} 32^{\prime} \mathrm{N}\right.$ and $\left.23^{\circ} 31^{\prime} \mathrm{E}\right)$, West Bengal, India. Root tips from the germinating seeds were pretreated with $4 \mathrm{mM} 8$-hydroxyquinoline at $14^{\circ} \mathrm{C}$ for $5 \mathrm{~h}$. Root tips were then fixed in acetic acid and methanol $(1: 3)$ overnight. Fixed root tips were digested with an enzyme mixture containing 1\% cellulase (Onozuka-RS, Sigma), $0.5 \%$ pectolyase (Sigma), and $0.75 \%$ macerozyme (Himedia) in a sodium citrate buffer at $37^{\circ} \mathrm{C}$ for $60 \mathrm{~min}$. The digested root tips were carefully washed with the same buffer twice and placed on a clean slide. After adding one drop of the freshly prepared fixative, the root tip was broken down into small pieces and air-dried for $90 \mathrm{~min}$ before staining. Chromosomes were stained with $2 \%$ Giemsa for $5 \mathrm{~min}$ and observed under a microscope. For 4',6-diamidino-2-phenylindole (DAPI) staining, the slides were preincubated in McIlvine buffer ( $\mathrm{pH} 7.0$ ), followed by staining with $0.2 \mu \mathrm{g} \mathrm{mL}^{-1}$ DAPI for $20 \mathrm{~min}$ in the dark and then observed under the fluorescent microscope Zeiss Axio Scope A1 equipped with a UV filter cassette. Photomicrographs were taken with an AxioCam ICc 5 and ZEN application suite.

The prepared slides needed to be aged for 10 days for Giemsa C-banding. Slides were first incubated in 5\% barium hydroxide at room temperature. After rinsing, they were placed in a copulin jar containing $2 \times \mathrm{SSC} \mathrm{pH}$ 7 for $40-60 \mathrm{~min}$ at $60^{\circ} \mathrm{C}$. Slides were stained with $2 \%$ Giemsa for $2 \mathrm{~min}$ and analyzed under a microscope. Individual chromosomes were measured with AxioVision 4.9.1 and categorized based on the arm ratio following Levan et al. (1964).
Results

\section{Karyotype analysis}

Karyomorphological studies in $N$. sativa were carried out in root tip cells of the germinating seeds and found to possess $2 n=12$ chromosomes. The application of cell wall digestive enzymes in chromosome preparation helps to spread every chromosome with a cytoplasmfree background so that details of structural features are revealed after Giemsa staining. The good resolution of the chromosome spread allows the measuring by the Axiocam application tool and marking of the different chromosomal attributes (Fig. 1A). The chromosomes are long, ranges from $11.25 \pm 0.07$ to $13.60 \pm 0.41 \mu \mathrm{m}$, except one chromosome pair that is short $(6.70 \pm 0.32 \mu \mathrm{m})$. The measurement of chromosomes along with their length has been presented in Table 1. Out of six chromosomal pairs, five large pairs are metacentric, and the small pair is subtelocentric. Thus, the diploid karyotype formula of $N$ sativa is $10 \mathrm{~m}+2$ st. The 2 nd pair possesses a subterminal secondary constriction, followed by a terminal knoblike structure. The 4th pair has a terminal secondary constriction and satellite (Fig. 1B, C).

\section{Fluorescent staining and Giemsa C-banding}

Fluorescent staining with DAPI reveals none of the chromosomes having DAPI-bands (Fig. 2A). The subterminal secondary constriction along with the terminal knob-like structure in the 2nd pair of chromosomes was identified. On the other hand, C-bands were found in every chromosome as well as in the nucleus (Fig. 2B). Two different types of C-bands polymorphism in terms of the amount and distribution pattern have been observed in the same population (Fig. 2C, E). The amount and pattern of distribution have been summarized in Table 2. Ideograms of these two types of polymorphism are also represented (Fig. 2D, F). In the first type (type I, Fig. 2C, D), all of the chromosomes show centromeric $\mathrm{C}$-bands. The rest of the C-bands are distributed mainly in the telomeric, subtelomeric, and pericentric regions. The 2nd pair has terminal C-bands with different sizes $(1.13 \mu \mathrm{m}$ and $1.35 \mu \mathrm{m})$ and intercalary bands near the centromere $(0.44 \mu \mathrm{m}$ and $0.46 \mu \mathrm{m})$. In the 3rd pair, the C-band $(0.83 \mu \mathrm{m})$ is in the subterminal position but only presents in a single chromosome of the homologous pair. The smallest pair has a prominent $\mathrm{C}$-band all over its length in the short arm and hence it was not possible to differentiate the bands from the centromere.

In the second type (type II, Fig. 2E, F), similar to the first one, all of the chromosomes have centromeric C-bands, and only three pairs of chromosomes contain the C-bands in the chromosomal arm. Although the distribution of the C-bands is nearly similar, it possesses another kind of polymorphism. The terminal band $(0.44 \mu \mathrm{m}$ and $0.40 \mu \mathrm{m})$ of the second chromosome pair is less intense than the type I plate, whereas the intercalary 


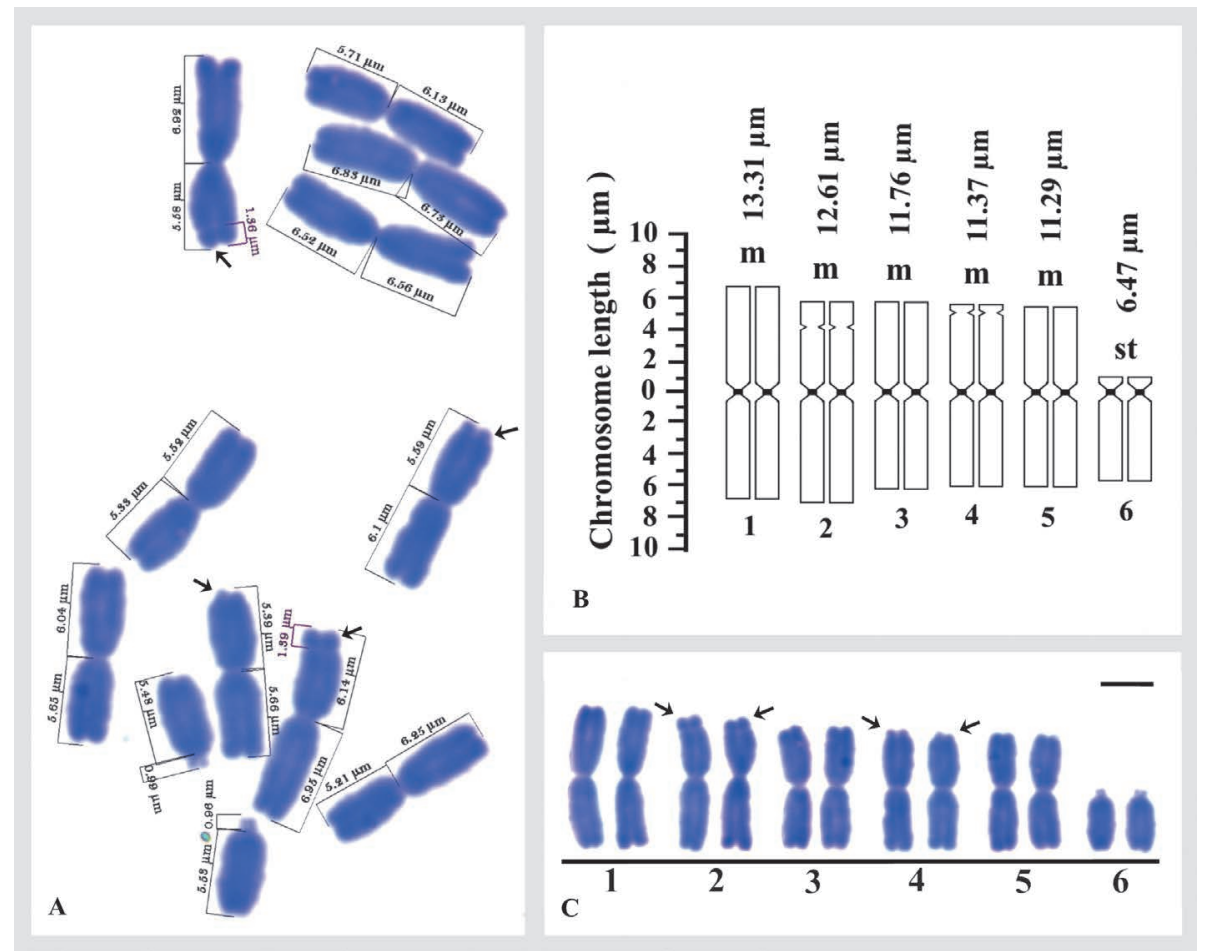

Fig. 1. Somatic metaphase chromosomes of $N$. sativa showing $2 n=12$ chromosomes. A: Giemsa staining of chromosomes including their size. Arrows indicate satellite and knob-like structures. B and C: Idiogram and karyogram representation of plate A. Scale bars $=5 \mu \mathrm{m}$.

Table 1. Chromosome parameters of Nigella sativa.

\begin{tabular}{ccccc}
\hline \hline Chromosome number & $\mathrm{S}(\mu \mathrm{m})$ & $\mathrm{L}(\mu \mathrm{m})$ & Total arm length $(\mu \mathrm{m})$ & Arm ratio $(\mathrm{L} / \mathrm{S})$ \\
\hline 1 & $6.73 \pm 0.16$ & $6.87 \pm 0.25$ & $13.60 \pm 0.41$ & 1.02 \\
2 & $5.84 \pm 2.19$ & $6.69 \pm 0.34$ & $12.53 \pm 0.12$ & 1.14 \\
3 & $5.63 \pm 0.07$ & $6.33 \pm 0.35$ & $11.95 \pm 0.27$ & 1.12 \\
4 & $5.46 \pm 0.04$ & $6.06 \pm 0.25$ & $11.52 \pm 0.21$ & $\mathrm{~m}$ \\
5 & $5.49 \pm 0.16$ & $5.76 \pm 0.23$ & $11.25 \pm 0.07$ & $\mathrm{~m}$ \\
6 & $1.22 \pm 0.35$ & $5.48 \pm 0.04$ & $6.70 \pm 0.32$ & $\mathrm{~m}$ \\
$\mathrm{st}$ & & 4.49 \\
\hline
\end{tabular}

$\mathrm{S}=$ short $\operatorname{arm}, \mathrm{L}=$ long $\operatorname{arm}, \mathrm{m}=$ metacentric, $\mathrm{st}=$ subtelocentric

band $(0.44 \mu \mathrm{m}$ and $0.47 \mu \mathrm{m})$ near the centromere is rather prominent. Another interesting polymorphism found in the 3rd pair is that there is a variable size of C-bands between two homologous chromosomes. The nucleus of the particular type also exhibits the same amount of variation in the interphase (Fig. 2B). The small pair of type II has an indiscriminate $\mathrm{C}$-band in its short arm and in the centromere, similar to that in type I. The polymorphic pattern of C-band distribution of types I and II have been presented in idiograms (Fig. 2D, F).

\section{Discussion}

Chromosomes of $N$. sativa are comparatively long, fewer in number, and have a good stainable property similar to the other members of the genus (Datta and Saha 2003). Out of six pairs, only one pair of small subtelocentric chromosomes that is present in this species is also found to be present in other species of the genus (Gilot-Delhalle et al. 1976). The number of secondary constrictions in $N$. sativa was controversial in the earlier reports. Ghosh and Datta (2006) reported that only the largest pair of the species was associated with a secondary constriction, whereas Datta and Biswas (1983) mentioned the presence of secondary constrictions in two pairs; this finding is similar to that in the present study. In enzymatic digestion of the cell wall coupled with Giemsa staining, the chromosome characters can be resolved more accurately. Thus, in the present observation, it can be stated that the second pair is associated with a secondary constriction in the subterminal position, whereas in the 4th pair, the secondary constriction is near the terminal position (Fig. 1B). Secondary constrictions in more than one pair have also been found in other Nigella species, e.g., N. damasence and N. orientalis (Gilot-Delhalle et al. 1976, Datta and Saha 2003, Hizume et al. 1982, 2013). The number and position of NORs can thus serve as a landmark for the identification of a particular chromosome.

The Giemsa C-banding method allows identifying the 

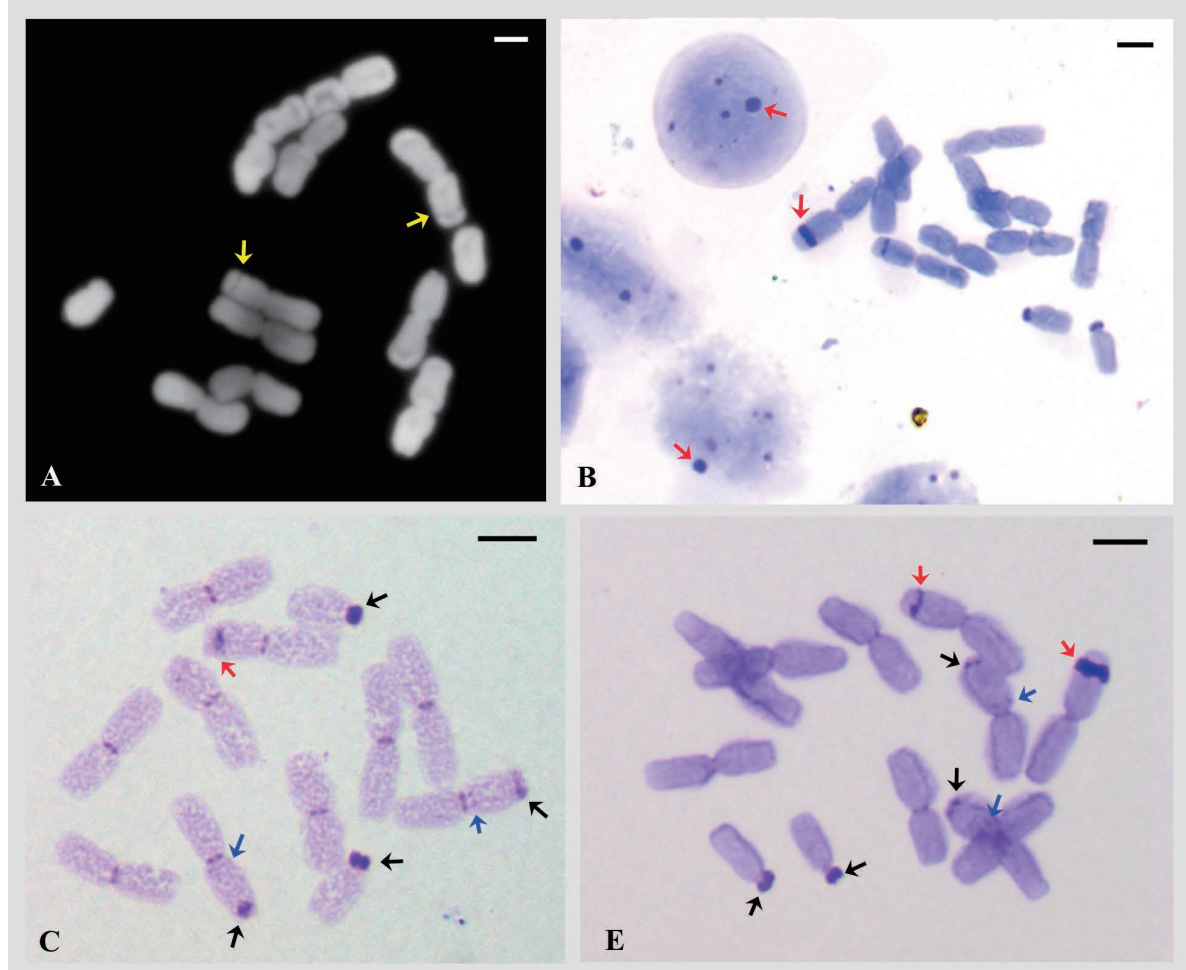

C
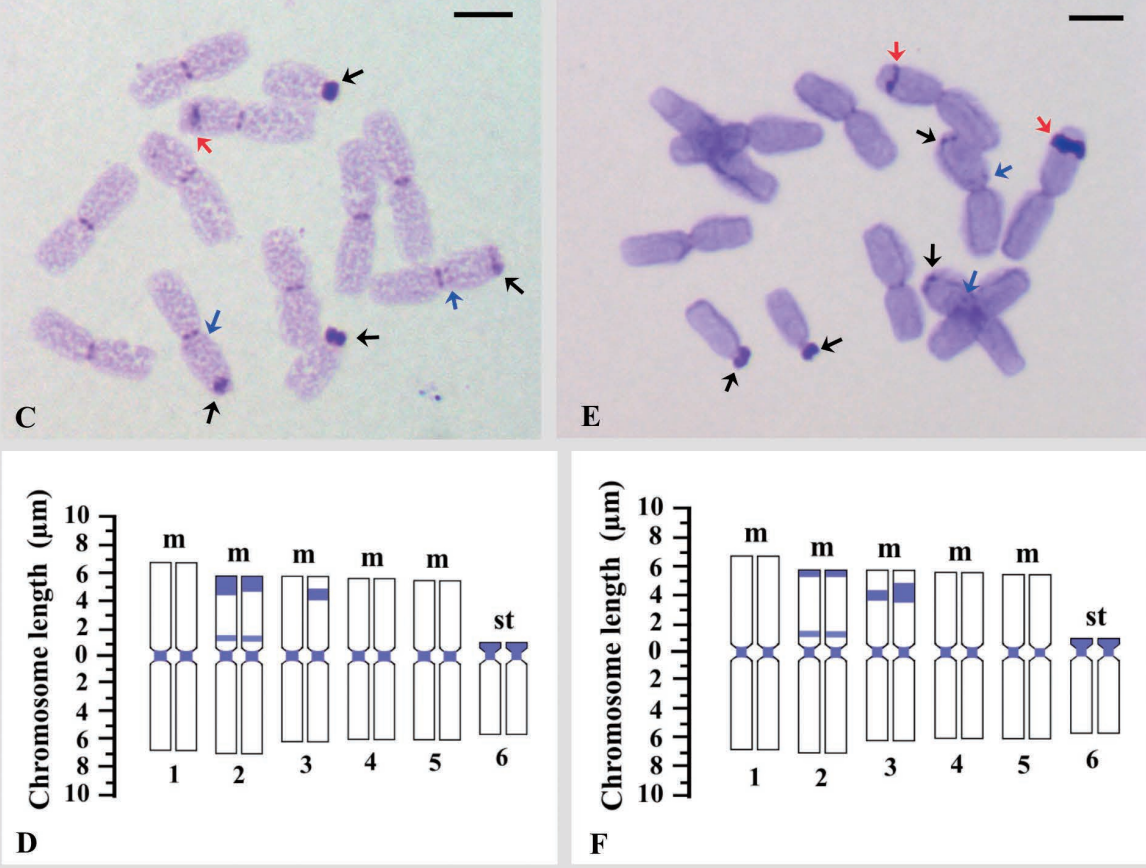

Fig. 2. DAPI staining and Giemsa C-banding polymorphism of metaphase chromosomes in $N$. sative. A: DAPI staining. Arrow indicates satellite chromosomes. B: Presence of constitutive heterochromatin bands in nucleus and chromosomes. Arrows indicate the large band correspondingly. C and E: chromosome showing polymorphic heterochromatin distribution in type-I and type-II individual respectively. Black arrows indicate terminal bands, red arrows indicate subterminal bands and blue arrows indicate intercalary bands. D and F: Representation of ideogram of type-I and type-II individual respectively along with their band distribution pattern. In $\mathrm{D}$, the subterminal band is present in one chromosome of third homologous pair while the band is absent in another chromosome. Scale bars $=5 \mu \mathrm{m}$.

Table 2. Distribution of constitutive heterochromatin in different chromosomes of two types of individuals.

\begin{tabular}{|c|c|c|c|c|c|c|c|c|}
\hline \multirow[b]{2}{*}{$\begin{array}{c}\text { Chromosome } \\
\text { number }\end{array}$} & \multicolumn{4}{|c|}{ Type-I individuals } & \multicolumn{4}{|c|}{ Type-II individuals } \\
\hline & $\begin{array}{c}\text { Centromeric } \\
\text { band (ranges } \\
0.4-0.6 \mu \mathrm{m})\end{array}$ & $\begin{array}{l}\text { Intercalary } \\
\text { band } \\
(\mu \mathrm{m})\end{array}$ & $\begin{array}{l}\text { Subterminal } \\
\text { band } \\
(\mu \mathrm{m})\end{array}$ & $\begin{array}{c}\text { Terminal } \\
\text { band } \\
(\mu \mathrm{m})\end{array}$ & $\begin{array}{c}\text { Centromeric } \\
\text { band (ranges } \\
0.4-0.6 \mu \mathrm{m})\end{array}$ & $\begin{array}{c}\text { Intercalary } \\
\text { band } \\
(\mu \mathrm{m})\end{array}$ & $\begin{array}{l}\text { Subterminal } \\
\text { band } \\
(\mu \mathrm{m})\end{array}$ & $\begin{array}{c}\text { Terminal } \\
\text { band } \\
(\mu \mathrm{m})\end{array}$ \\
\hline 1 & + & - & - & - & + & - & - & - \\
\hline 2 & + & - & - & - & + & - & - & - \\
\hline 3 & + & 0.46 & - & 1.35 & + & 0.44 & - & 0.44 \\
\hline 4 & + & 0.44 & - & 1.13 & + & 0.47 & - & 0.43 \\
\hline 5 & + & - & 0.83 & - & + & - & 0.73 & - \\
\hline 6 & + & - & - & - & + & - & 1.36 & - \\
\hline 7 & + & - & - & - & + & - & - & - \\
\hline 8 & + & - & - & - & + & - & - & - \\
\hline 9 & + & - & - & - & + & - & - & - \\
\hline 10 & + & - & - & - & + & - & - & - \\
\hline 11 & + & - & - & 1.46 & + & - & - & 1.21 \\
\hline 12 & + & - & - & 1.48 & + & - & - & 1.34 \\
\hline
\end{tabular}


constitutive heterochromatin distribution in chromosomes, which proves advantageous in karyosystematic studies (Giuseppina et al. 2010). Apart from cytotaxonomy, the distribution of heterochromatin can also help unknot the evolutionary complexity at least for closely related species (Siljak-Yakovlev 2017). Polymorphism, or the variation in constitutive heterochromatin amount and location at the intraspecific level or in a population, has been considered to play a significant role in its dissimilar vegetative and sexual characters as well as in ecological adaption (Vosa 1996, 1997). In the present study with $N$. sativa, the location of the constitutive heterochromatin or C-bands in the chromosomes shows considerable similarity with the other species of the genus Nigella (GilotDelhalle et al. 1976, Marks 1977). Two different types of C-band polymorphism have been found (Table 2) mainly based on the amount and existence of the constitutive heterochromatin. The location of the bands on different chromosomes is much conserved, and no variation has been detected in this context in any of the individuals. Evidence from several other plant species supported that the drastic change in the constitutive heterochromatin pattern is rare between related groups although it can vary in its amount (Vosa 1973, Greilhuber and Speta 1978, Miranda et al. 1997, Guerra 2000). The occurrence of constitutive heterochromatin polymorphism diversity in a single population is probably related to the pollination choice of the species. Naturally, in a freemating or cross-pollinating population, the intraspecific or even intraindividual heteromorphism of the heterochromatin content is much more frequent (Georgiou et al. 1992, Winterfeld and Roser 2007). In a type I individual, the 3 rd pair shows a heteromorphism between homologous chromosomes. One chromosome of the $3 \mathrm{rd}$ pair (Fig. 2D) is associated with the subterminal C-band, whereas the other did not. This may a typical instance of the cross-pollination event where two homologues come from two different parents in the same population. Similarly, the quantitative variation of constitutive heterochromatin contents has been noticed in the 3rd pair of type II individuals (Fig. 2F). In addition, variation in terminal heterochromatin amount in the 2nd pair of type I and type II individuals have been noticed. This too supports the fact that random polymorphism can take place in different individuals of a population and can be dispersed through the sexual reproduction process. Dissimilarity in constitutive heterochromatin content has been reported earlier in the other member, $N$. hispanica, of the genus but not in this species (Gilot-Delhalle et al. 1976). Moreover, the precise chromosomal dataset in the present study is very significant in breeding process to further improve this commercially important plant.

\section{Acknowledgements}

All the authors acknowledge Swami Kamalasthana- nda, Principal, Ramakrishna Mission Vivekananda Centenary College, Rahara, Kolkata, India for the facilities provided for this study. Further, the authors are thankful to Md. Salim for providing the seeds.

\section{References}

Blakey, D. H. and Vosa, C. G. 1981. Heterochromatin and chromosome variation in cultivated species of Tulipa subg. Eriostemones (Liliaceae). Plant Syst. Evol. 139: 47-55.

Datta, A. K. and Biswas, A. K. 1983. Karyotype analysis in four Nigella species. Cell Chromosome Res. 6: 21-24.

Datta, A. K. and Saha, A. 2003. Cytomorphological studies and seed protein characterization of Nigella sativa L. and Nigella damascena L. Cytologia 68: 51-60.

Dávila-Rodríguez, M. I., Gutiérrez, E. C., Flores, R. C., Pita, M., Fernández, J. L., López-Fernández, C. and Gosálvez, J. 2011. Constitutive heterochromatin polymorphisms in human chromosomes identified by whole comparative genomic hybridization. Eur. J. Histochem. 55: e28.

Davison, J., Tyagi, A. and Comai, L. 2007. Large-scale polymorphism of heterochromatic repeats in the DNA of Arabidopsis thaliana. BMC Plant Biol. 7: 44.

Dubey, P. N., Singh, B., Mishra, B. K., Kant, K. and Solanki, R. K. 2016. Nigella (Nigella sativa L.): A high value seed spice with immense medicinal potential. Ind. J. Agric. Sci. 86: 967-979.

Gatti, M. and Pimpinelli, S. 1992. Functional elements in Drosophila melanogaster heterochromatin. Annu. Rev. Genet. 26: 239-276.

Georgiou, A., Karatagilis, S. and Raupakias, D. 1992. Inter- and intraplant C-banding polymorphism in one population of Aegilops comosa var. comosa (Poaceae). Plant Syst. Evol. 180: 105-114.

Ghosh, A. and Datta, A. K. 2006. Karyotyping of Nigella sativa L. (black cumin) and Nigella damascena L. (love-in-a-mist) by image analyzing system. Cytologia 71: 1-4.

Gilot-Delhalle, J., Degrave, N. and Moutschen, J. 1976. Cytotaxonomic investigation of the genus Nigella (Helleboreae) with Cbanding techniques. Caryologia 29: 139-154.

Giuseppina, B., Brullo, C., Pulvirenti, S., Scrugli, A., Terrasi, M. C. and D'Emerico, S. 2010. Advances in chromosomal studies in Neottieae (Orchidaceae): Constitutive heterochromatin, chromosomal rearrangements and speciation. Caryologia 63: 184-191.

Greilhuber, J. and Speta, F. 1978. Quantitative analyses of C-banded karyotypes, and systematics in the cultivated species of the Scilla siberica group (Liliaceae). Plant Syst. Evol. 129: 63-109.

Guerra, M. 2000. Patterns of heterochromatin distribution in plant chromosomes. Genet. Mol. Biol. 23: 1029-1041.

Haque, S. M., Paul, S. and Ghosh, B. 2016. Karyological studies of two hot chilli pepper cultivars from two different geographical regions of India: Bhut jolokia, Capsicum chinense Jacq. and Bullet Lanka, Capsicum annuum L. Nucleus 59: 227-233.

Hizume, M., Shiraishi, H., Matsusaki, Y. and Shibata, F. 2013. Localization of $45 \mathrm{~S}$ and $5 \mathrm{~S}$ rDNA on chromosomes of Nigella damascena, Ranunculaceae. Cytologia 78: 379-381.

Hizume, M., Tanaka, A. and Shigematsu, H. 1982. Detection of nucleolar organizing regions in the chromosomes of Nigella damacena. Experientia 38: 238-239.

Jabeen, R., Iftikhar, T., Mengal, T. and Iqbal, K. M. 2012. A comparative chromosomal count and morphological karyotyping of three indigenous cultivars of Kalongi (Nigella sativa L.). Pak. J. Bot. 44: 1007-1012.

John, B. and Miklos, G. L. G. 1979. Functional Aspects of Satellite DNA and Heterochromatin. In: Bourne, G. H., Danelli, J. F. and Jeon, K. W. (eds.). International Review of Cytology Vol. 58. Academic Press, New York. pp. 1-114.

John, B. 1981. Heterochromatin variation in natural populations. 
Chromosomes Today 7: 128-137.

John, B. 1988. The Biology of Heterochromatin. In: Verma, R. S. (ed.). Heterochromatin: Molecular and Structural Aspects. Cambridge University, Cambridge. pp. 1-147.

Khan, A., Chen, H. C., Tania, M. and Zhang, D. Z. 2011. Anticancer activities of Nigella sativa (black cumin). Afr. J. Tradit. Complement Altern. Med. 8 Suppl: 226-232.

Levan, A., Fredga, K. and Sandberg, A. A. 1964. Nomenclature for centromeric position on chromosomes. Hereditas 52: 201-220.

Majdalawieh, A. F. and Fayyad, M. W. 2016. Recent advances on the anti-cancer properties of Nigella sativa, a widely used food additive. J. Ayurveda Integr. Med. 7: 173-180.

Marks, G. E. 1977. The nature of centromeric dots in Nigella chromosomes. Chromosoma 62: 369-373.

Miranda, M., Ikeda, F., Endo, T., Moriguchi, T. and Omura, M. 1997. Comparative analysis on the distribution of heterochromatin in Citrus, Poncirus and Fortunella chromosomes. Chromosome Res. 5: 86-92.

Schwarzacher, T. and Schweizer, D. 1982. Karyotype analysis and heterochromatin differentiation with Giemsa C-banding and fluorescent counterstaining in Cephalanthera (Orchidaceae). Plant Syst. Evol. 141: 91-113.

Shaker, S. S., Mohammadi, A. and Shahli, M. K. 2017. Cytological studies on some ecotypes of Nigella sativa L. in Iran. Cytologia 82: 123-126.

Siljak-Yakovlev, S., Godelle, B., Zoldos, V., Vallès, J., Garnatje, T. and
Hidalgo, O. 2017. Evolutionary implications of heterochromatin and rDNA in chromosome number and genome size changes during dysploidy: A case study in Reichardia genus. PLoS One 12: e0182318.

Sybenga, J. 1992. Cytogenetics in genetics and plant breeding. Springer-Verlag, Berlin/Heidelberg.

Verma, R. S. 1998. Heterochromatin: Evolutionary Aspects. In: Verma, R. S. (ed.). Advances in Genome Biology Vol. 5. Elsevier, Amsterdam. pp. 497-504.

Vosa, C. G. and Marchi, P. 1972. Quinacrine fluorescence and Giemsa staining in plants. Nat. New Biol. 237: 191-192.

Vosa, C. G. 1973. Heterochromatin recognition and analysis of chromosome variation in Scilla sibirica. Chromosoma 43: 269-278.

Vosa, C. G. 1976. Heterochromatic banding patterns in Allium. II. Heterochromatin variation in species of the Paniculatum group. Chromosoma 57: 119-133.

Vosa, C. G. 1996. Some aspects of karyotype evolution in Liliflorae: Heterochromatin variation and ecology in Allium pulchellum. Bocconia 5: 267-270.

Vosa, C. G. 1997. Heterochromatin and ecological adaptation in Southern African Ornithogalum (Liliaceae). Caryologia 50: 97-103.

Winterfeld, G. and Röser, M. 2007. Chromosomal localization and evolution of satellite DNAs and heterochromatin in grasses (Poaceae), especially tribe Aveneae. Plant Syst. Evol. 264: 75-100. 